\title{
Sailing for Rehabilitation of Patients with Severe Mental Disorders: Results of a Cross Over Randomized Controlled Trial
}

\author{
Mauro G. Carta ${ }^{1, *}$, Federica Maggiani ${ }^{1}$, Laura Pilutzu ${ }^{1}$, Maria F. Moro ${ }^{1}$, Gioia Mura ${ }^{1}$, \\ Federica Cadoni ${ }^{1}$, Federica Sancassiani ${ }^{1}$, Marcello Vellante ${ }^{1}$, Sergio Machado ${ }^{2}$ and Antonio Preti ${ }^{1}$
}

\author{
${ }^{I}$ Department of Public Health and Clinical and Molecular Medicine University of Cagliari and Centro di Psichiatria di \\ Consulenza e Psicosomatica AUOC Cagliari, Cagliari, Italy; ${ }^{2}$ Institute de Psychiatry of Federal University of Rio de \\ Janeiro (IPUB/UFRJ)
}

\begin{abstract}
This study set out to evaluate the effectiveness of a sailing and learning-to-sail rehabilitation protocol in a sample of patients diagnosed with severe mental disorders. The study was a randomized, crossover, waiting-list controlled trial, following recruitment in the Departments of Mental Health of South Sardinia. Participants were outpatients diagnosed with severe mental disorders, recruited through announcements to the directors of the Departments of Mental Health of South Sardinia. Out of the 40 patients enrolled in the study, those exposed to rehabilitation with sailing during a series of guided and supervised sea expeditions near the beach of Cagliari (Sardinia), where the aim to explore the marine environment while sailing was emphasized, showed a statistically significant improvement of their clinical status (measured by BPRS) and, as well, of their general functioning (measured by HoNOS Scale) against the control group. The improvement was maintained at follow-up for some months only: after 12 months, the patients returned to their baseline values on the measures of psychopathology and showed a worsening trend of their quality of life. Sailing can represent a substitute of important experiences that the patients with severe mental disorders miss because of their illness.
\end{abstract}

Keywords: Bipolar disorder, quality of life, rehabilitation, sailing, Schizophrenia.

\section{INTRODUCTION}

Severe mental disorders impact heavily on the life of the affected patients and their families, and bear huge direct and indirect costs on society at large [1-3].

Lost opportunities, such as the early discontinuation of school and the deprivation of experiences as a consequence of the early onset of a severe mental disorder, negatively affect patients' quality of life [4]. Rehabilitation can improve the social outcome of patients, well beyond the effectiveness of the prescribed therapeutic interventions [5, 6]. Sports activities have often been used as a rehabilitation tool for patients with severe psychiatric disorders [7, 8]. Different sports can enhance important aspects that are useful in rehabilitation, such as improving self-esteem and selfconfidence, participation in group dynamics and improvement of communication skills, best knowledge and awareness of the body [9-11].

It is well-known that "participation in sailing by people with disabilities is widely regarded as having positive outcomes on self-esteem" and the general health of the participants [12]. Many anecdotal experiences are reported in the literature on the use of sailing in the rehabilitation of disabled people [13] as well in behavioral disorders [14]. A few studies have tried to measure this effectiveness by experimental designs of adapted programs of sailing for people

*Address correspondence to this author at the Centro di Psichiatria di Consulenza e Psicosomatica AUOC Cagliari and University of Cagliari, Italy; Tel: + 335 499994; Fax: +39 070 6093498; E-mail: mgcarta@tiscali.it with physical disabilities [15]. Learning to sail and sailing have been used in the rehabilitation practice of some Public Mental Health Centers in Italy (e.g., Genoa and Trieste) with people suffering of severe mental disorders, but the efficacy of these programs was never measured. Both the Trieste and the Genoa Mental Health Sailing Programs are so popular that each year they take part in international boat races, in particular the "Barcolana Race" in Trieste, where the crew of "Matti per la Vela" (Crazy for sailing), composed of patients of the Genoa Centre, was accompanied by the internationally renowned sailing champion Giovanni Soldini as their skipper; while the Trieste Team uses the "Moro di Venezia" sailing ship that raced in the America's Cup in the past [16]. Therefore the assignment of stimulating tasks like sailing to achieve higher quality-of-life goals is a well known albeit poorly studied practice. In fact so far no studies have been carried out with standardized tools and experimental designs to measure the potential benefits of a program where sailing is the rehabilitation means for patients with severe chronic psychiatric disorders. This study was conceived to study how much learning to sail and sailing may impact on psychopathological residual symptoms, improve social skills, selfesteem and the perceived quality of life, with a cross-over randomized trial versus placebo, in a group of patients with severe chronic mental diseases. During the boat trips for practice, organized as a motivation stimulus to keep on sailing, we used a boat equipped with sonar to identify marine mammals; the crew was also involved in monitoring the sonar. Our intent was in fact to dampen the competitive aspect related to learning with a view to participating to races and the related stress [17], and emphasize the pleasure of "going 
to sea". In this case, learning to stay on a boat and sail was stimulated by the fact that the patients were working on a boat equipped to identify fish and cetaceans in the Gulf of Cagliari.

\section{METHODS}

The data were collected as part of a study aimed at assessing a multi-modal protocol of rehabilitation sponsored by the Regional Authority of Sardinia. The board of the Planning Center of the Regional Authority of Sardinia as well as the Institutional review board of the Università Europea del Mediterraneo ONLUS approved the study project. The study protocol complied with the revisions held in Tokyo 2004 of the Declaration of Helsinki of 1995. Informed consent have been obtained from the invited participants.

\section{Study Sample and Design}

Design: Randomized, controlled, crossover clinical trial. Each subject was randomized to this sequence of treatments:

a) Adjunctive therapy plus rehabilitation with sailing plus current drug treatment (first year) and adjunctive therapy with a traditional rehabilitation program (self-help group discussion and work therapy) plus current drug treatment (second year); or b) adjunctive therapy with the traditional rehabilitation program (self-help group discussion and work therapy) plus current drug treatment (first year) and adjunctive therapy with rehabilitation with sailing plus current drug treatment (second year). The study was run from May 2010 to October 2010 (6 months of sailing rehabilitation in Group A), followed by 6 months of no expeditions in the open sea (from November 2010 to April 2011); the crossover was run from May 2011 to October 2011 (6 months of sailing rehabilitation in Group B). Participants: The sample included 40 patients with a diagnosis of Schizophrenia, affective psychoses, or severe personality disorders according to ICD10 criteria (WHO 1990). The inclusion criterion was to have been in charge of a mental health care network for no less than 2 years and to have a psychopathological condition in clinical remission, with no episodes of crisis in the past four months. Patients were recruited through announcements to the directors of the Departments of Mental Health of South Sardinia (Italy) in March and April 2010. The sample was extracted from an initial pool of 53 patients: $13(24.5 \%)$ patients were excluded because they were not fit to go into the open sea since they suffered from severe obesity, motor troubles, or seasickness. Patients provided their written informed consent to participate. Field operations were conducted in the Gulf of Cagliari. Patients were randomized to two groups $(\mathrm{A}, \mathrm{B})$ that would be exposed to rehabilitation with sailing in the open sea in different periods. Randomization was by coin toss, and made by two clinicians who did not participate in the study. The allocation sequence was kept in sealed envelopes until treatments were assigned, which occurred after baseline evaluation. The clinical and functional status of patients was rated in a day-care center where the patients were exposed to the multi-modal rehabilitation treatment. Raters were blind to the treatment allocation of the patients as far as rehabilitation with sailing was concerned. However, since patients often talked of their experience in the open sea, blinding was difficult to preserve.

\section{Intervention}

The rehabilitation protocol included monthly group meetings, weekly social skills training and leisure sessions on a sailing ship, and a cycle of lessons about sea life. The protocol included a program of bi-monthly expeditions to the open sea on board of a sailing boat. Patients were instructed on ship management and on the use of the ship's hydrophone. A hydrophone is a microphone used underwater to record or listen to underwater sounds. All submarine sounds are captured, and researchers need to detect the different sources in order to understand the movements of shoals of cetaceans. After each expedition, patients had the opportunity of discussing their impressions and feelings about the experience. Each expedition took about 5 hours. During the 6 months of the open sea expeditions, all patients continued to attend the day-care center located next to the port of $\mathrm{Ca}$ gliari ("Centro di Marina Piccola"), but only those in the active group participated in the expeditions and in the lesson program that was performed in the same center; the control group was involved in a more traditional rehabilitation activity characterized by self-help groups and group-support work in the garden.

\section{Assessment}

The clinical status of the patients was measured at monthly intervals with the Health of the Nation Outcome Scale (HoNOS), to assess behavioral problems and disability [18, 19], and the 24-item Brief Psychiatric Rating Scale (BPRS), to assess general psychopathology [20, 21].

Every three months since the baseline patients were also evaluated with the "Global Assessment of Functioning" [22].

All raters $(n=3)$ were trained in the administration of the assessment tools, and all had minimum two years' professional experience with patients diagnosed with severe mental disorders. In the sample, intra-class correlation (ICC) coefficient at baseline for HoNOS was .61 (95\% confidence of interval: .42 to .77 ), and it was .87 (.80 to .92) for the BPRS, ICC did not change in repeated assessments over time. Testretest stability was measured from baseline to 3 months on the GAF: ICC was .96 (.94 to .98).

\section{Statistics}

Data were analyzed with the Statistical Package for Social Science (SPSS) for Windows (Chicago, Illinois60606, USA), version 17. Due to the multiple testing the tests have been two-tailed; the threshold for significance has been fixed at $p<.0001$; according to Bayesian interpretations of having an highest chance of replication [23].

Inter-group comparisons of nominal data have been carried out with chi square test, or Fisher's exact test, if request.

Sample homogeneity in numerical data were measured by means of " $t$ " Student's tests for mean differences.

Differences by time have been measured by means of a general linear model (GLM) accounting for the repeated observations.

Student paired t-test was used to assess differences over time on assessment measures at pre-defined time points: from baseline to the 6th month (corresponding to the begin- 
ning and end of the rehabilitation with sailing in Group A), and from the 12th to the 18th month (corresponding to the beginning and end of the rehabilitation with sailing in Group $\mathrm{B})$. The reliable change index (RCI) was used to provide a standardized score measured as the change in the score of a participant from pre-intervention to post-intervention measurement, independent from error measurement. The RCI is a measure of significant clinical change [24]. The difference between post and pre intervention is divided by the standard error of the difference of the two test scores:

$\mathrm{RCI}=(\mathrm{x} 2-\mathrm{x} 1) /$ Sdiff. When RCI is found to be greater of 1.96, the post-test score reflects the real change (Jacobson et al. 1984). No data were missing in the database. An independent research assistant rechecked a sub-sample of the data $(20 \%)$ after they were entered: the error rate was lower than $1 \%$.

\section{RESULTS}

The sample included 40 patients, 38 males and 2 females, with an age range from 23 to 58 years (mean age $=38.5$ years, $\mathrm{SD}=9.1$ ). A very limited fraction of patients continued education after compulsory school, most were unmarried with a disability pension, and a large majority of them were living with someone else taking care of him/her (Table 1). The sample included $23(57.5 \%)$ patients diagnosed with a schizophrenia spectrum psychosis, $11(27.5 \%)$ patients diagnosed with an affective psychosis (most, $\mathrm{n}=6$, had bipolar disorder co-morbid with alcohol or substance abuse), and 6 $(15 \%)$ patients diagnosed with a personality disorder (principally, borderline personality disorder).

No statistically significant differences have been found between the two groups regarding major socio-demographic variables and the clinical measures at baseline but age, with the patients in Group B being on average and marginally younger than those in Group A (Table 1). Long-term effects of the rehabilitation with sailing Group A recorded 2 dropouts, one in the second month and the other in the third. Group B had 5 dropouts, all occurring in the 13th month (one month after the start of the rehabilitation with sailing for this group). Dropouts did not differ from the patients who stayed in treatment by any of the measures at baseline ( $p>.05$ in all comparisons). A repeated ANOVA measurement with a Greenhouse-Geisser correction determined that measures of psychopathology underwent statistically significant changes over time. Mean HoNOS and BPRS scores differed significantly statistically between time points, and the changes differed between groups (Table 2). Betweengroup differences over time were less evident on the GAF. As far as the measure of quality of life is concerned, differences between groups over time were evident for the physical and social areas, and less evident for the psychological and environmental areas (Table 2). Comparisons at the predefined time points showed that changes over time were congruent with an effect of the rehabilitation with sailing, with improvement on the HoNOS and the BPRS in the groups in coincidence with the expeditions in the open sea, and no changes when there were no expeditions (Fig. 1). Differences across the period of exposition to the rehabilitation with sailing were more evident in Group B as far as symptoms and social functioning were concerned, and in Group A concerning quality of life. 15 patients out of 18
(83\%) in Group A showed RCI on the HoNOS, versus 3 out of $20(15 \%)$ in Group B at the end of the first year $\left(\chi^{2}=15.11, \mathrm{df}=1, \mathrm{p}<.0001\right)$. The proportion reversed in the second year: 2 patients out of $18(11 \%)$ vs 14 out of 15 $(93 \%)$ in the second year $\left(\chi^{2}=18.97, \mathrm{df}=1, \mathrm{p}<.0001\right)$. Patients with RCI on the BPRS were 16 out of $18(89 \%)$ in Group A and 2 out of $20(10 \%)$ in Group B after the first year $\left(\chi^{2}=20.59, \mathrm{df}=1, \mathrm{p}<.0001\right)$; they were 2 out of 18 $(11 \%)$ in Group A and 15 out of $15(100 \%)$ in Group B at the end of the second year $\left(\chi^{2}=22.44\right.$, df $\left.=1, p<.0001\right)$. Concerning the GAF, RCI for improvement was observed in 13 out of $18(72 \%)$ patients in Group A and in no Group B patient $\left(\chi^{2}=18.86, \mathrm{df}=1, \mathrm{p}<.0001\right)$ at the end of the first year; and in 6 out of $18(33 \%)$ in Group A and in 15 out of $15(100 \%)$ in Group B at the end of the second year $\left(\chi^{2}=12.96, \mathrm{df}=1, \mathrm{p}<.0001\right)$. The benefit of the rehabilitation with sailing did not persist at follow-up (see Fig. 1). In Group A, this was observed 6 months after the end of the rehabilitation with sailing through the scores on the HoNOS, the BPRS, and the GAF. The 6-month follow-up after termination of rehabilitation with sailing in Group B was not assessed because the study was not allocated funds to complete the second phase of the cross-over trial.

\section{DISCUSSION}

The patients exposed to "rehabilitation with sailing" during a series of guided and supervised sea expeditions near the beach of Cagliari (Sardinia), showed a statistically significant improvement of their clinical status and of their general functioning. The improvement was maintained at follow-up for some months only: after 12 months the patients returned to their baseline values on pretty all measures of psychopathology, and showed a trend for a worsening of social functioning. Overall, the patients with severe mental disorders who received rehabilitation with sailing as an adjunctive treatment to a multi-modal protocol of rehabilitation showed a psychopathological improvement on both the BPRS and the HoNOS. Global functionality as measured by the GAF also improved. Controls with pharmacotherapy and traditional rehabilitation programs did not improve their BPRS, HoNOS, or GAF scores during the experimental period of the crossover trial. Thus rehabilitation with sailing seems a good adjunctive treatment of patients with severe mental disorders, but the improvement is limited to the period of exposition. Although sailing is thought useful in improving the self-esteem and general health of the participants with physical disabilities and behavioral disorders [12, 14], to our knowledge this is the first study that used randomized controlled cross-over and standardized measures of outcome to evaluate the effectiveness of a rehabilitation period on a sailing boat for people with serious mental problems. Although the data are to be considered preliminary, given the small sample used, and need therefore to be confirmed, they look very promising especially when compared with other rehabilitation programs. It should be underlined that at the same time as this study, our group had been following for about a year a cohort of 300 users of the public mental health services in Sardinia, this cohort having similar clinical characteristics to the sample of this study [14]. In this study the HoNOS score did not improve in most patients of the centers participating in the study during the 12-month follow-up, with the sole exception of the patients of those centers that 
Table 1. Baseline socio-demographic and clinical characteristics of the patients enrolled in the study.

\begin{tabular}{|c|c|c|c|}
\hline All data: no. (\%) or mean (SD) median & $\begin{array}{c}\text { Group A } \\
\mathbf{n}=\mathbf{2 0}\end{array}$ & $\begin{array}{c}\text { Group B } \\
\mathbf{n}=\mathbf{2 0}\end{array}$ & Statistics \\
\hline $\begin{array}{c}\text { Sex } \\
\text { Males } \\
\text { Females }\end{array}$ & $\begin{array}{c}18(90 \%) \\
2(10 \%)\end{array}$ & $\begin{array}{c}20(100 \%) \\
0(0 \%)\end{array}$ & Chi-Square $=0.52, \mathrm{df}=1, \mathrm{p}=.46$ \\
\hline Age & $41.5(8.3) 41$ & $35.5(9.0) 31$ & $\mathrm{t}=2.18, \mathrm{df}=38, \mathrm{p}=.03$ \\
\hline $\begin{array}{l}\text { Civil status } \\
\text { Unmarried } \\
\text { Divorced }\end{array}$ & $\begin{array}{c}19(95 \%) \\
1(5 \%)\end{array}$ & $\begin{array}{c}20(100 \%) \\
0(0 \%)\end{array}$ & Chi-Square $=0.00, \mathrm{df}=1, \mathrm{p}=1.00$ \\
\hline $\begin{array}{c}\text { Occupational status } \\
\text { Employed } \\
\text { Unemployed } \\
\text { Disability pension }\end{array}$ & $\begin{array}{c}1(5 \%) \\
7(35 \%) \\
12(60 \%)\end{array}$ & $\begin{array}{c}0(0 \%) \\
4(20 \%) \\
16(80 \%)\end{array}$ & Chi-Square $=2.39, \mathrm{df}=2, \mathrm{p}=.30$ \\
\hline $\begin{array}{c}\text { Diagnosis } \\
\text { Schizophreniaspectrum psychoses } \\
\text { Affective psychoses } \\
\text { Personality disorders }\end{array}$ & $\begin{array}{l}14(70 \%) \\
4(20 \%) \\
2(10 \%)\end{array}$ & $\begin{array}{l}9(45 \%) \\
7(35 \%) \\
4(20 \%)\end{array}$ & Chi-Square $=2.57, \mathrm{df}=2, \mathrm{p}=.27$ \\
\hline \multicolumn{4}{|l|}{ Clinical variables } \\
\hline
\end{tabular}

Group A refers to the group that participated in the dolphin-finding expeditions in the first year of the study; Group B is the group that participated in the dolphin-finding expeditions in the second year of the study, and served as a control group of Group A.

Table 2. Changes over time in measures of psychopathology, social functioning and quality of life.

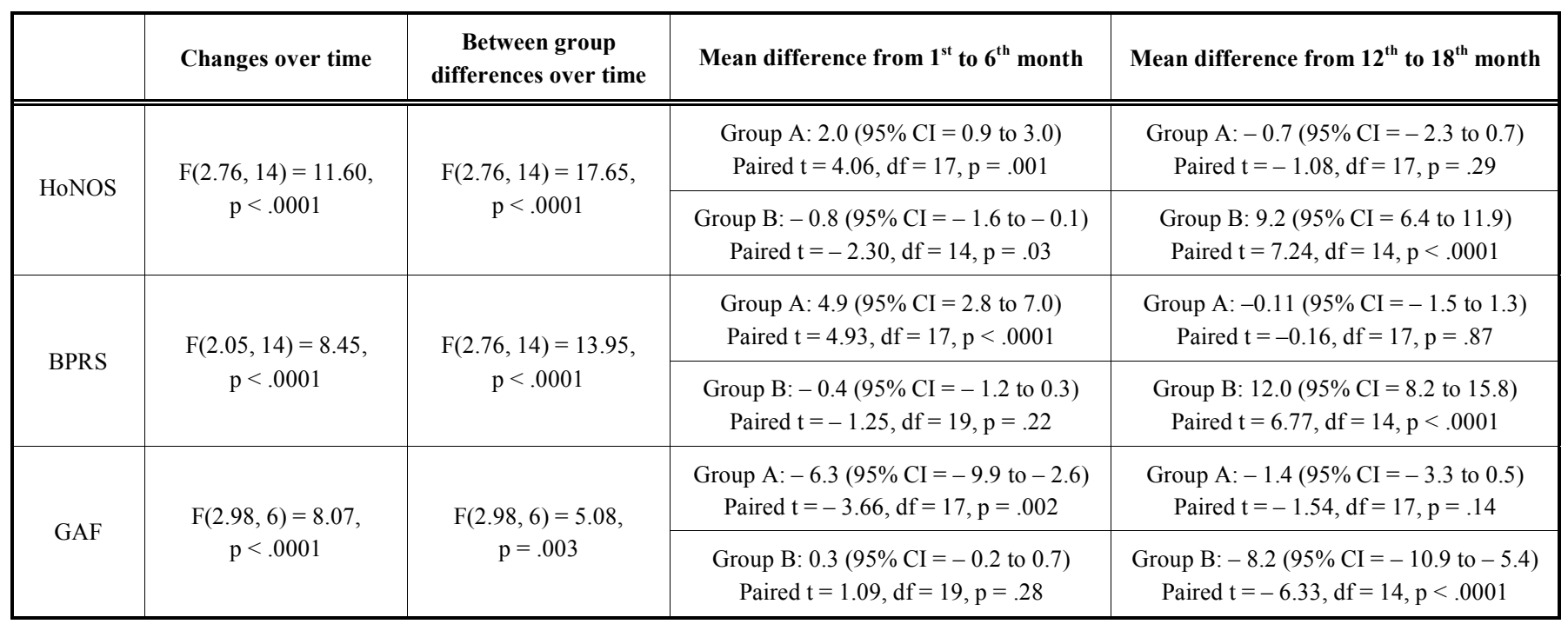

In group A patients sailed from the $1^{\text {st }}$ month to the $6^{\text {th }}$ month In group B patients sailed from the $12^{\text {th }}$ month to the $18^{\text {th }}$ month 

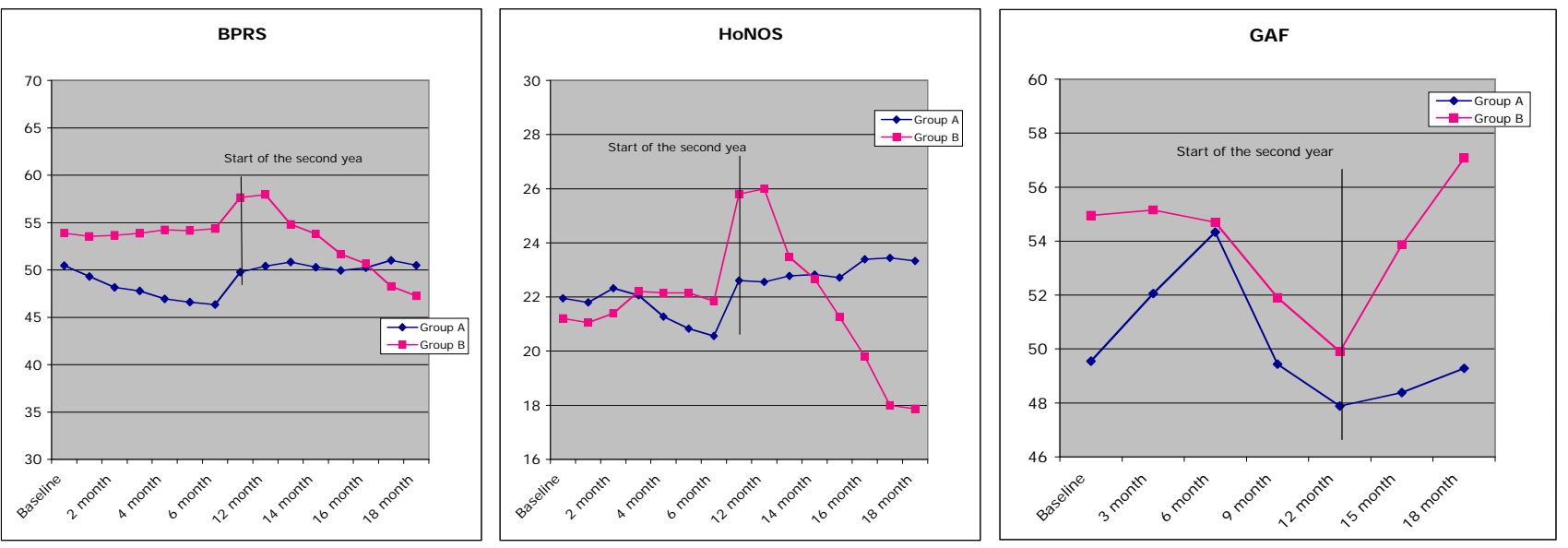

Fig. (1). Distribution of scores on the BPRS, HoNOS and GAF over time, by group.

had hired more staff. However these exceptions related to two small towns that, at the beginning of the study, showed patients with the worst scores, probably because of their limited human resources. In this study, the HoNOS score improves only in the six months following the intervention. This suggests a general confirmation that a longer rehabilitation activity may significantly improve the outcome. The last follow-up study did not show any improvement in the severity of the symptoms over time; in fact no statistically significant changes were shown in the CGI score [25] measuring clinical severity. Routine care activities may improve the scores of the scales relating to disability, but do not seem to affect the patient's symptoms, while a special program like sailing does. In this study also the BPRS score measuring psychopathology was shown to improve in the experimental group against the control group. More interesting observations about the outcome were also obtained comparing the results of a group of patients who were following a special job placement project in the field of renewable energy [26]. In the three-month follow-up of this trial, the comparison between the subjects who were in the employment project and the control subjects in routine rehabilitation programs, revealed an improvement in HoNOS total score in both groups with non-significant differences between groups over time. Greater improvement was shown only in the HoNOS "impairment" scale for the people in the job program. However when comparing subjects in the work placement program with a control group that was on drugs only (without any rehabilitation program), both groups showed an improvement in the HoNOS total score, with a greater improvement in the job placement group. On the contrary, in this study the Honos total score improved significantly in the sailing group against the group with rehabilitation routine activity. This might suggest some benefits in terms of motivation and rehabilitation generated by a sailing project. It should however be pointed out first that this study had a longer duration (six months versus three months), and secondly, that an employment project in a competitive environment may lead to higher long-term results since it gives patients an active work task. The two approaches could become complementary if the design of the sailing project is confirmed to have a greater potential to stimulate motivation and autonomy. Unlike a workplace study, the present study did not analyze specifically which aspects of the HoNOS scale had improved and did not make any comparison with patients receiving routine drug treatment without rehabilitation activities. In the light of the findings of the study on work inclusion, this type of analysis might be of interest. A new approach to the concept of recovery from schizophrenia and other serious mental illnesses is pushing clinicians and rehabilitation professionals to a more optimistic attitude that involves more patients in rehabilitation pathways [27-29]. This approach sees recovery more like a customized path, rather than the outcome of a rigid and pre-packaged rehabilitation plan. That is, a project that can give hope, help create an affective base and achieve safe, supportive relationships, empowerment, social inclusion, coping skills [30, 31]. The recovery of a solid sense of self (that the psychotic disorder has broken or destroyed) has been proposed as the main element of this approach [32]. It has been suggested that the process of creating a positive sense of the self can be facilitated by experiences of interpersonal acceptance, mutuality, a sense of belonging to a social group, by developing skills and compete with tasks that one cannot gradually solve [3335]. It is easily understood that to know how to hang the sail offers elements that may facilitate the recovery path; for example, working in a team with experts stimulates a sense of belonging and helps people learn interesting things; it is also linked to a new sense of autonomy and freedom (sail to the sea) in an environment full of new and unusual stimuli (the sound of marine mammals and fish). In fact an item that may have enhanced motivation, and therefore cannot be generalized to all studies on the use of sailing in rehabilitation, is the care given by this program to the aspects of going by boat to explore the marine and underwater life. The intervention described in this study is of a complex type indeed. What are the real, effective factors in the complex intervention analyzed in this study is hard to understand, as is the possibility to use a single factor alone. It should be pointed out that the program involved multiple new experiences for the subjects, such as governing a boat, experiencing community life on the boat, scanning for dolphins, learning how to use hydrophones, and others. Some concern was raised on the cost of similar programs and on the possibility to generalize them, since the same criticism was previously raised against similar studies in the form of cruises by boat organized to experience closeness with cetaceans [36]. The overall cost of this project was of 120,000 Euro for the two years. The costs 
were inclusive of the boat costs; of two full-time two-year grants, one assigned to a skipper researcher and the other to a rehabilitation therapist researcher; of 2 part-time assistant experts, an aid skipper and a psychologist; and of the insurance costs for the patients and the researchers. Overall, the cost was around 3,000 Euro per patient. The expected cost without the experimental design is about $30 \%$ of the aboverated amount, because no control group support would be required, as would not the measurement of the intervention with research tools and the preparation of the intervention i.e., to process data in autumn and winter. The expected cost of the intervention without any research design in the actual health-care setting would be around 800 Euro per patient. Generally, this study seems to suggest the need to identify motivating objectives to pique and support rehabilitation programs, and to improve the quality of life in people suffering from severe psychiatric disorders. In this sense a captivating experience may interrupt the evolution to chronicity, which is sometimes favored by the scarcity of stimuli and impaired quality of life $[37,38]$

\section{LIMITATIONS}

One of the limits of this study is the small sample size, but the crossover model has some advantages against a longitudinal non-crossover model [39]. Mainly because in a crossover study each subject serves as his own control, thus the influence of confounding factors is smaller than in a longitudinal model. Also must consider that crossover designs are powerfully efficient and therefore require a fewer sample size than longitudinal designs [39]. Thus the design partially counteracts the limit of sample size. Several additional limitations must be borne in mind. Since this was a pilot study, the sample was small, so no multivariate analysis could be carried out. The assessors did not remain blind to the treatment status of the participants, because patients talked enthusiastically about their experience. Moreover, patients might have described themselves more favorably in the period of expeditions to avoid being withdrawn from the study because of relapse into an active episode of their illness.

\section{CONCLUSION}

This is the first study to show that rehabilitation with sailing may improve the clinical status of patients diagnosed with severe mental disorders, and impact positively on their global functioning and quality of life as well. In all likelihood, a program grounded on learning how to govern a sailing vessel might be interesting enough and capture the attention of the patients to favor greater effectiveness of standard rehabilitation protocols, but this should be specifically tested.

\section{CONFLICT OF INTEREST}

The authors confirm that this article content has no conflicts of interest.

\section{ACKNOWLEDGEMENTS}

\section{Dr FM and Dr LP}

This study received financial support by European Social Found 2007-2013 (Grant no. CRP3_165). No other financial support were received for this study.

\section{Study Design: MGC, FM and LP}

Data Collection and Analysis: FM, LP, FC, MV, AP, GM, MFM, FS and MGC

Manuscript Preparation: MGC, MFM, AP, FM, LP, FC, SM and CD

\section{REFERENCES}

[1] McEvoy JP. The costs of schizophrenia. J Clin Psychiatry 2007; 68 (Suppl 14): 4-7.

[2] Keck PE Jr, Kessler RC, Ross R. Clinical and economic effects of unrecognized or inadequately treated bipolar disorder. J Psychiatr Pract 2008; 14 (Suppl 2): 31-8.

[3] Angst J, Meyer TD, Adolfsson R, et al. Hypomania: a transcultural perspective. World Psychiatry 2010 Feb; 9(1): 41-9.

[4] Mura G, Petretto DR, Krishna MB, Carta MG. Schizophrenia: from epidemiology to rehabilitation. Clin Pract Epidemiol Ment Health 2012; 8: 22-7.

[5] Carta MG, Angermeyer MC, Sancassiani F, et al. A follow-up on patients with severe mental disorders in Sardinia after two changes in regional policies: poor resources still correlate with poor outcomes. BMC Psychiatry 2013; 13(1): 333. doi: 10.1186/1471244X-13-333

[6] Carta MG, Agaj A, Harapej E, et al. Outcomes of discharged females versus those waiting for discharge from Vlore Psychiatric Hospital (Albania). Int J Soc Psychiatry 2013; 59(7): 682-9.

[7] Hardoy MC, Seruis ML, Floris F, et al. Benefits of exercise with mini tennis in intellectual disabilities: effects on body image and psychopathology. Clin Pract Epidemiol Ment Health 2011; 7: 15760 .

[8] Mura G, Moro MF, Patten SB, Carta MG. Exercise as an add-on strategy for the treatment of major depressive disorder: a systematic review. CNS Spectr 2014 Mar 3: 1-13. [Epub ahead of print]

[9] Carta MG, Hardoy MC, Pilu A, et al. Improving physical quality of life with group physical activity in the adjunctive treatment of major depressive disorder. Clin Pract Epidemiol Ment Health 2008; 26: 1 .

[10] Pilu A, Sorba M, Hardoy MC, et al. Efficacy of physical activity in the adjunctive treatment of major depressive disorders: preliminary results. Clin Pract Epidemiol Ment Health 2007; 3: 8.

[11] Mura G, Sancassiani F, Migliaccio GM, Collu G, Carta MG. The association between different kinds of exercise and quality of life in the long term. results of a randomized controlled trial on the elderly. Clin Pract Epidemiol Ment Health 2014; 10: 36-41.

[12] Recio AC, Becker D, Morgan M, Saunders NR, Schramm LP, McDonald JW 3rd. Use of a virtual reality physical ride-on sailing simulator as a rehabilitation tool for recreational sports and community reintegration: a pilot study. Am J Phys Med Rehabil 2013; 92(12): 1104-9. doi: 10.1097/PHM.0000000000000012

[13] Zimmermann R, Brendler R. Sailing with the handicapped. Rehabilitation (Stuttg) 1983; 22(4): 166-8.

[14] Hegemann T. Rehabilitative success of a social therapy sailing project. Prax Kinderpsychol Kind Kratz G, Söderback I, Guidetti S, Hultling C, Rykatkin T, Söderström M. Prax Kinderpsychol Kinderpsychiatr 1991; 40(2): 61-6.

[15] Kratz G, Söderback I, Guidetti S, Hultling C, Rykatkin T, Söderström M. Wheelchair users' experience of non-adapted and adapted clothes during sailing, quad rugby or wheel-walking. Disabil Rehabil 1997; 19(1): 26-34.

[16] Mantini R, 2006. Available from: http://www.triestesalutementale.it/ segnalazioni/dsm/2006/2006-09-25_barcolana.htm

[17] Rodek J, Idrizović K, Zenić N, Perasović B, Kondric M. Differential analysis of the doping behaviour templates in three types of sports. Coll Antropol 2013; 37 (Suppl 2): 211-7.

[18] Wing JK, Beevor AS, Curtis RH, Park BG, Hadden S, Burns A. Health of the Nation Outcome Scales (HoNOS): research and development. Br J Psychiatry 1998; 172: 11-8.

[19] Parabiaghi A, Barbato A, D'Avanzo B, Erlicher A, Lora A. Assessing reliable and clinically significant change on Health of the Nation Outcome Scales: method for displaying longitudinal data. Aust NZ J Psychiatry 2005; 39: 719-25. 
[20] Overall JE, Gorham DE. The brief psychiatric rating scale. Psychol Rep 1962; 10: 799-812.

[21] Roncone R, Ventura J, Impallomeni M, et al. Reliability of an Italian standardized and expanded Brief Psychiatric Rating Scale (BPRS 4.0) in raters with high vs. low clinical experience. Acta Psychiatr Scand 1999; 100: 229-36.

[22] Moos RH, McCoy L, Moos BS. Global assessment of functioning (GAF) ratings: determinants and roles as predictors of one-year treatment outcomes. J Clin Psychol 2000; 56: 449-61.

[23] Katki HA. Invited commentary: evidence-based evaluation of $\mathrm{p}$ values and Bayes factor. Am J Epidemiol 2008; 168: 384-8.

[24] Jacobson NS, Foilette WC, Revenstorf D. Psychotherapy outcome research: methods for reporting variability and evaluating clinical significance. Behav Ther 1984; 15: 336-52.

[25] Guy W. The Clinical Global Impression. In: ECDEU Assessment Manual for Psychopharmacology (revised). Rockville MD: US Department of Health Education and Welfare 1976: 217-22.

[26] Carta M, Sancassiani F, Lecca M, et al. Coping with the crisis: people with severe mental disorders acting for social change through sustainable energy. Clin Pract Epidemiol Ment Health 2013; 9: 214-20.

[27] Silverstein SM, Bellack AS. A scientific agenda for the concept of recovery as it applies to Schizophrenia. Clin Psychol Rev 2008; 28: 1108-24.

[28] Nordén T, Malm U, Norlander T. Resource Group Assertive Community Treatment (RACT) as a tool of empowerment for clients with severe mental illness a meta-analysis. Clin Pract Epidemiol Ment Health 2012; 8: 144-51.

[29] Miyamoto Y, Sono T. Lessons from peer support among individuals with mental health difficulties a review of the literature. Clin Pract Epidemiol Ment Health 2012; 8: 22-9.
[30] Ramon S, Healy B, Renouf N. Recovery from mental illness as an emergent concept and practice in Australia and the UK. Int J Soc Psychiatry 2007; 53(2): 108-22.

[31] Drukker M, Visser E, Sytema S, van Os J. Flexible assertive community treatment, severity of symptoms and psychiatric health service use, a real life observational study. Clin Pract Epidemiol Ment Health 2013; 9: 202-9.

[32] Sells DJ, Stayner DA, Davidson L. Recovering the self in Schizophrenia: an integrative review of qualitative studies. Psychiatr Q 2004; 75(1): 87-97.

[33] Flammer E, Steinert T, Eisele F, Bergk J, Uhlmann C. Who is subjected to coercive measures as a psychiatric inpatient? A multilevel analysis. Clin Pract Epidemiol Ment Health 2013; 9: 110-9.

[34] Dunn EC, Wewiorski NJ, Rogers ES. The meaning and importance of employment to people in recovery from serious mental illness: results of a qualitative strudy. Psychiatr Rehabil J 2008; 32: 59-62.

[35] Carta MG, Balestrieri M, Murru A, Hardoy MC. Adjustment disorder: epidemiology, diagnosis and treatment. Clin Pract Epidemiol Ment Health 2009; 5: 15.

[36] Basil B, Mathews M. Human and animal health: strengthening the link: methodological concerns about animal facilitated therapy with dolphins. BMJ 2005; 331: 1407

[37] Mantovani G, Astara G, Lampis B, et al. Impact of psychosocial interventions on quality of life of elderly cancer patients. Psychooncology 1996; 5: 127-35.

[38] Mantovani G, Astara G, Lampis B, et al. Evaluation by multidimensional instruments of heath-related quality of life of elderly cancer patients undergoing three different "psychosocial" treatment approaches. A randomized clinical trial. Supportive Care Cancer 1996; 4: 129-40.

[39] Jone BK, Michael G. Design and Analysis of Cross-Over Trials (2nd ed.). London: Chapman and Hall 2003.

(C) Carta et al.; Licensee Bentham Open.

This is an open access article licensed under the terms of the Creative Commons Attribution Non-Commercial License (http://creativecommons.org/licenses/by-nc/3.0/) which permits unrestricted, non-commercial use, distribution and reproduction in any medium, provided the work is properly cited. 